\title{
New criteria for stability of neutral differential equations with variable delays by fixed points method
}

\author{
Dianli Zhao ${ }^{1,2}$
}

Correspondence:

Tc_zhaodianli@139.com

${ }^{1}$ College of Science, University of

Shanghai for Science and

Technology, Shanghai, 200093,

China

Full list of author information is

available at the end of the article

\begin{abstract}
The linear neutral differential equation with variable delays is considered in this article. New criteria for asymptotic stability of the zero solution are established using the fixed point method and the differential inequality techniques. By employing an auxiliary function on the contraction condition, the results of this article extend and improve previously known results. The method used in this article can also be used for studying the decay rates of the solutions.
\end{abstract}

Keywords: fixed points, stability, neutral differential equation, variable delays

\section{Introduction}

The objective of this article is to investigate the stability of the zero solution of the first-order linear neutral differential equations with variable delays

$$
x^{\prime}(t)=-b(t) x(t-\tau(t))+c(t) x^{\prime}(t-\tau(t))
$$

and it's generalized form

$$
x^{\prime}(t)=-a(t) x(t)-\sum_{j=1}^{N} b_{j}(t) g\left(x\left(t-\tau_{j}(t)\right)\right)+\sum_{j=1}^{M} c_{j}(t) x^{\prime}\left(t-\tau_{j}(t)\right)
$$

by fixed point method under assumptions: $a, b, c, b_{j}, c_{j} \in C\left(R^{+}, R\right), \tau, \tau_{j} \in C\left(R^{+}, R^{+}\right)$, $t-\tau(t) \rightarrow \infty$ and $t-\tau_{j}(t) \rightarrow \infty$ as $t \rightarrow \infty$.

Recently, Burton and others [1-10] applied fixed point theory to study stability. It has been shown that many of problems encountered in the study of stability by means of the Lyapunov's direct method can be solved by means of the fixed point theory. Then, together with Sakthivel and Luo [11,12] investigate the asymptotic stability of the nonlinear impulsive stochastic differential equations and the impulsive stochastic partial differential equations with infinite delays by means of the fixed point theory. On the other hand, Luo $[13,14]$ firstly considers the exponential stability for stochastic partial differential equations with delays by the fixed point method. Zhou and Zhong [15] study the exponential $p$-stability of neutral stochastic differential equations with multiple delays. Pinto and Seplveda [16] talk about $H$-asymptotic stability by the fixed point method in neutral nonlinear differential equations with delay. By the same method, Equation 1 and its generalization have been investigated by many authors. For

(c) 2011 Zhao; licensee Springer. This is an Open Access article distributed under the terms of the Creative Commons Attribution License (http://creativecommons.org/licenses/by/2.0), which permits unrestricted use, distribution, and reproduction in any medium, provided the original work is properly cited. 
example, Raffoul [17] and Jin and Luo [18] have studied the equation

$$
x^{\prime}(t)=-a(t) x(t)-b(t) x(t-\tau(t))+c(t) x^{\prime}(t-\tau(t))
$$

and give the following result.

Theorem A (Raffoul [17]) Let $\tau(t)$ be twice differentiable and $\tau^{\prime}(t) \neq 1$ for all $t \in R$. Suppose that there exists a constant $\alpha \in(0,1)$ such that for $t \geq 0$

$$
\int_{0}^{t} a(u) d u \rightarrow \infty \text { as } t \rightarrow \infty
$$

and

$$
\left|\frac{c(t)}{1-\tau^{\prime}(t)}\right|+\int_{0}^{t} e^{-\int_{s}^{t} a(u) d u}\left|b(s)+\frac{\left[a(s) c(s)+c^{\prime}(s)\right]\left(1-\tau^{\prime}(s)\right)+c(s) \tau^{\prime \prime}(s)}{\left(1-\tau^{\prime}(s)\right)^{2}}\right| d s \leq \alpha
$$

Then every solution $x(t)=x(t, 0, \psi)$ of (1) with a small continuous initial function $\psi(t)$ is bounded and tends to zero as $t \rightarrow \infty$.

Theorem B (Jin and Luo [18]) Let $\tau(t)$ be twice differentiable and $\tau^{\prime}(t) \neq 1$ for all $t$ $\in R$. Suppose that there exists a constant $0<\alpha<1$ and a continuous function $h: R^{+} \rightarrow$ $R$ such that for $t \geq 0$,

$$
\liminf _{t \rightarrow \infty} \int_{0}^{t} h(s) d s>-\infty
$$

and

$$
\begin{aligned}
& \left|\frac{c(t)}{1-\tau^{\prime}(t)}\right|+\int_{t-\tau(t)}^{t}|h(s)-a(s)| d s+\int_{0}^{t} e^{-\int_{s}^{t} h(u) d u} \mid-b(s)+[h(s-\tau(s))-a(s-\tau(s))] \\
& \cdot\left(1-\tau^{\prime}(s)\right)-r(s)\left|d s+\int_{0}^{t} e^{-\int_{s}^{t} h(u) d u}\right| h(s) \mid\left(\int_{s-\tau(s)}^{s}|h(u)-a(u)| d u\right) d s \leq \alpha,
\end{aligned}
$$

where $r(s)=\frac{\left(h(s) c(s)+c^{\prime}(s)\right)\left(1-\tau^{\prime}(s)\right)+c(s) \tau^{\prime \prime}(s)}{\left(1-\tau^{\prime}(s)\right)^{2}}$.

Then the zero solution of (1) is asymptotically stable if and only if $\int_{0}^{t} h(u) d u \rightarrow \infty$ as $t \rightarrow \infty$.

Ardjouni and Djoudi [19] study the generalized linear neutral differential equation of the form

$$
x^{\prime}(t)=-\sum_{j=1}^{N} b_{j}(t) x\left(t-\tau_{j}(t)\right)+\sum_{j=1}^{N} c_{j}(t) x^{\prime}\left(t-\tau_{j}(t)\right) .
$$

Theorem C (Ardjouni and Djoudi [19]) Let $\tau_{j}(t)$ be twice differentiable and $\tau_{j}{ }^{\prime}(t) \neq 1$ for all $t \in\left[m_{j}(\sigma), \infty\right)$. Suppose that there exist constant $0<\alpha<1$ and continuous functions $h_{j}:\left[m_{j}(\sigma), \infty\right) \rightarrow R$ such that for $t \geq 0$,

$$
\liminf _{t \rightarrow \infty} \int_{0}^{t} H(s) d s>-\infty,
$$


and

$$
\begin{aligned}
& \sum_{j=1}^{N}\left|\frac{c_{j}(t)}{1-\tau^{\prime}{ }_{j}(t)}\right|+\sum_{j=1}^{N} \int_{t-\tau_{i}(t)}^{t} \mid h_{j}(s) d s \\
& +\sum_{j=1}^{N} \int_{0}^{t} e^{-\int_{s}^{t} H(u) d u}\left|-b_{j}(s)+\left[h_{j}\left(s-\tau_{j}(t)\right)\right]\left(1-\tau^{\prime}{ }_{j}(s)\right)-r_{j}(s)\right| d s \\
& +\sum_{j=1}^{N} \int_{0}^{t} e^{-\int_{s}^{t} H(u) d u}|H(s)|\left(\int_{s-\tau_{i}(s)}^{s}\left|h_{j}(u)\right| d u\right) d s \leq \alpha,
\end{aligned}
$$

where $H(t)=\sum_{j=1}^{N} h_{j}(t)$, and $r_{j}(t)=\frac{\left[H(t) c_{j}(t)+c_{j}^{\prime}(t)\right]\left(1-\tau_{j}^{\prime}(t)\right)+c_{j}(t) \tau_{j}^{\prime \prime}(t)}{\left(1-\tau^{\prime}(t)\right)^{2}}$. Then the zero solution of (4) is asymptotically stable if and only if $\int_{0}^{t} H(u) d u \rightarrow \infty$ ast $\rightarrow \infty$.

Obviously, Theorem B improves Theorem A. Theorem C extends Theorem B. Without the loss of generality, we denote $\frac{c_{1}(t)}{1-\tau^{\prime} 1(t)}=\frac{c(t)}{1-\tau^{\prime}(t)}$. The contraction conditions (3), (4) and (5) imply $\left|\frac{c(t)}{1-\tau^{\prime}(t)}\right|<\alpha$ for some constant $\alpha \in(0,1)$, and hence Theorems A, B, and $C$ will be all invalid if $\left|\frac{c(t)}{1-\tau^{\prime}(t)}\right|<1$ does not hold. In this article, we first give some criteria for asymptotic stability by fixed points method that can be applied to neutral equation which does not satisfy the constraint $\left|\frac{c(t)}{1-\tau^{\prime}(t)}\right|<1$. Furthermore, the method used in this article can also be used to study the decay rates of the solutions which has not been studied using the fixed point theory to the best of our knowledge except that the exponential stability has been discussed by Luo [13,14] and Zhou and Zhong [15].

This article is organized as follows: Section 2 includes some notations and definitions. In Section 3, the linear delay differential equations and its generalization are discussed by using the fixed points method. Sufficient conditions for asymptotical stability are presented. In Section 4, we present two examples to show applications of some obtained results. The last Section is the conclusion.

\section{Preliminary notes}

Let $R=(-\infty,+\infty), R^{+}=[0,+\infty)$ and $Z^{+}=1,2,3, \ldots$ and $C\left(S_{1}, S_{2}\right)$ denote the set of all continuous functions $\phi: S_{1} \rightarrow S_{2} . N, M \in Z^{+}$. For each $\sigma \in R^{+}$, define $m(\sigma)=\inf \{s-\tau(s): s \geq$ $\sigma\}, m_{j}(\sigma)=\inf \left\{s-\tau_{j}(s): s \geq \sigma\right\}, \bar{m}(\sigma)=\min \left\{m_{j}(\sigma), j=1,2, \ldots, N\right\}$ and $C(\sigma)=C([m(\sigma), \sigma]$, $R)$ with the supremum norm $\|\psi\|=\max \{|\psi(s)|: m(\sigma) \leq s \leq \sigma\}$. For each $(\sigma, \psi) \in R^{+} \times C$ $([m(\sigma), \sigma], R)$, a solution of (1) through $(\sigma, \psi)$ is a continuous function $x:[m(\sigma), \sigma+a) \rightarrow$ $R^{n}$ for some positive constant $a>0$ such that $x$ satisfies (1) on $[\sigma, \sigma+a)$ and $x=\psi$ on [m $(\sigma), \sigma]$. We denote such a solution by $x(t)=x(t, \sigma, \psi)$. For each $(\sigma, \psi) \in R^{+} \times C([m(\sigma)$, $\sigma], R)$, there exists a unique solution $x(t)=x(t, \sigma, \psi)$ of (1) defined on $[\sigma, \infty)$. Similarly, the solution of (2) can be defined.

Next, we state some definitions of the stability.

Definition 2.1. For any $\psi C(\sigma)$. The zero solution of (1) is said to be

(1) stable, if for any $\varepsilon>0$ and $\sigma \geq 0$, there exists $a \delta=\delta(\varepsilon, \sigma)>0$ such that $\psi \in C(\sigma)$ and $\|\psi\|<\delta$ imply $|x(t, \sigma, \psi)|<\varepsilon$ for $t \geq \sigma$;

(2) asymptotically stable, if $x(t, \sigma, \psi)$ is stable and for any $\varepsilon>0$ and $\sigma \geq 0$, there exists a $\delta=\delta(\varepsilon, \sigma)>0$ such that $\psi \in C(\sigma)$ and $\|\psi\|<\delta$ implies $\lim _{t \rightarrow \infty} x(t, \sigma, \psi)=0$. 
Definition 2.2. Assume that $\lambda(t) \rightarrow \infty$ as $t \rightarrow \infty$ and satisfies $\lambda(t+s) \leq \lambda(t) \lambda(s)$ for $t, s \in R^{+}$largely enough. Then for any $\psi \in C(\sigma)$, the zero solution of (1) is said to be $\lambda$-stable if $\limsup _{t \rightarrow \infty} \frac{\log |x(t)|}{\log \lambda(t)} \leq-\gamma$ for some constant $\gamma>0$.

Remark 1. In Definition 2.2,

(1) let $\lambda(t)=e^{t}$, we called the zero solution of (1) is exponentially stable.

(2) let $\lambda(t)=1+t$, we called the zero solution of (1) is polynomially stable.

(3) let $\lambda(t)=\log (1+t)$, we called the zero solution of (1) is logarithmically stable.

\section{Main results}

In this section, sufficient conditions for stability are presented by the fixed point theory. We first give some results on stability of the zero solution of Equation 1. Then, we generalized the results of the stability to Equation 2 .

Consider the first-order delay neutral differential equation of the form

$$
x^{\prime}(t)=-b(t) x(t-\tau(t))+c(t) x^{\prime}(t-\tau(t)) .
$$

Now, we state our main result in the following.

Theorem 3.1. Let $\tau(t)$ be twice differentiable and $\tau^{\prime}(t) \neq 1$ for all $t \in[m(\sigma), \infty)$. Suppose that

(i) there exists a continuous function $h:[m(\sigma), \infty) \rightarrow R$ satisfying $\lim _{t \rightarrow \infty} \int_{\sigma}^{t} h(u) d u=\infty$;

(ii) there exists a bounded function $p:[m(\sigma), \infty) \rightarrow(0, \infty)$ with $p(\sigma)=1$ such that $p^{\prime}(t)$ exists on $[m(\sigma), \infty)$;

(iii) there exists a constant $\alpha \in(0,1)$ such that for $t \geq \sigma$

$$
\begin{aligned}
& \left|\frac{p(t-\tau(t))}{p(t)} \frac{c(t)}{1-\tau^{\prime}(t)}\right|+\int_{t-\tau(t)}^{t}|h(s)| d s \\
& +\int_{\sigma}^{t} e^{-\int_{s}^{t} h(u) d u}\left|-\beta(s)+h(s-\tau(t))\left(1-\tau^{\prime}(s)\right)-r(s)\right| d s \\
& +\int_{\sigma}^{t} e^{-\int_{s}^{t} h(u) d u}|h(s)|\left(\int_{s-\tau(s)}^{s}|h(u)| d u\right) d s \leq \alpha,
\end{aligned}
$$

where

$$
\beta(t)=\frac{b(t) p(t-\tau(t))+c(t) p^{\prime}(t-\tau(t))-p^{\prime}(t)}{p(t)}
$$

and

$$
r(t)=\frac{h(t) c(t) p(t) p(t-\tau(t))+c^{\prime}(t) p(t-\tau(t))}{p^{2}(t)\left(1-\tau^{\prime}(t)\right)}+\frac{c(t) p(t-\tau(t)) \tau^{\prime \prime}(t)}{p(t)\left(1-\tau^{\prime}(t)\right)^{2}}-c(t) p^{\prime}(t-\tau(t)) .
$$

Then the zero solution of (1) is asymptotically stable.

Proof. Let $z(t)=\psi(t)$ on $[m(\sigma), \sigma]$ and for $t \geq \sigma$

$$
x(t)=p(t) z(t)
$$


Make substitution of (7) into (1) to show

$$
z^{\prime}(t)=-\frac{b(t) p(t-\tau(t))+c(t) p^{\prime}(t-\tau(t))-p^{\prime}(t)}{p(t)} z(t-\tau(t))+\frac{p(t-\tau(t))}{p(t)} c(t) z^{\prime}(t-\tau(t)) .
$$

Since $p(t)$ is bounded, it remains to prove that the zero solution of (8) is asymptotically stable.

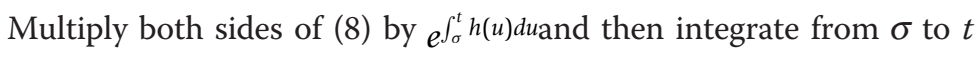

$$
\begin{aligned}
z(t)= & \psi(\sigma) e^{-\int_{\sigma}^{t} h(u) d u}+\int_{\sigma}^{t} e^{-\int_{s}^{t} h(u) d u} h(s) z(s) d s \\
& -\int_{\sigma}^{t} e^{-\int_{s}^{t} h(u) d u} \frac{b(s) p(s-\tau(s))+c(s) p^{\prime}(s-\tau(s))-p^{\prime}(s)}{p(s)} z(s-\tau(s)) d s \\
& +\int_{\sigma}^{t} e^{-\int_{s}^{t} h(u) d u} \frac{p(s-\tau(s))}{p(s)} c(s) z^{\prime}(s-\tau(s)) d s .
\end{aligned}
$$

Performing an integration by parts, we have

$$
\begin{aligned}
z(t)= & \psi(\sigma) e^{-\int_{\sigma}^{t} h(u) d u}+\int_{\sigma}^{t} e^{-\int_{s}^{t} h(u) d u} d\left(\int_{s-\tau(s)}^{s} h(u) x(u) d u\right) \\
& +\int_{\sigma}^{t} e^{-\int_{s}^{t} h(u) d u}\left\{-\frac{b(s) p(s-\tau(s))+c(s) p^{\prime}(s-\tau(s))-p^{\prime}(s)}{p(s)}+p(s-\tau(s))\left(1-\tau^{\prime}(s)\right)\right\} \\
& \times z(s-\tau(s)) d s+\int_{\sigma}^{t} e^{-\int_{s}^{t} h(u) d u} \frac{p(s-\tau(s))}{p(s)} \frac{c(s)}{1-\tau^{\prime}(s)} d z(s-\tau(s)) \\
= & \left(\psi(\sigma)-\frac{p(\sigma-\tau(\sigma))}{p(\sigma)} \frac{c(\sigma)}{1-\tau^{\prime}(\sigma)} \psi(\sigma-\tau(\sigma))-\int_{\sigma-\tau(\sigma)}^{\sigma} h(s) \psi(s) d s\right) e^{-\int_{\sigma}^{t} h(u) d u} \\
& +\frac{p(t-\tau(t))}{p(t)} \frac{c(t)}{1-\tau^{\prime}(t)} z(t-\tau(t))+\int_{t-\tau(t)}^{t} h(s) z(s) d s \\
& +\int_{\sigma}^{t} e^{-\int_{s}^{t} h(u) d u}\left(-\beta(s)+h(s-\tau(s))\left(1-\tau^{\prime}(s)\right)-r(s)\right) z(t-\tau(t)) d s \\
& -\int_{\sigma}^{t} e^{-\int_{s}^{t} h(u) d u} h(s)\left(\int_{s-\tau(s)}^{s} h(u) z(u) d u\right) d s
\end{aligned}
$$

where

$$
\beta(t)=\frac{b(t) p(t-\tau(t))+c(t) p^{\prime}(t-\tau(t))-p^{\prime}(t)}{p(t)}
$$

and

$$
r(t)=\frac{h(t) c(t) p(t) p(t-\tau(t))+c^{\prime}(t) p(t-\tau(t))}{p^{2}(t)\left(1-\tau^{\prime}(t)\right)}+\frac{c(t) p(t-\tau(t)) \tau^{\prime \prime}(t)}{p(t)\left(1-\tau^{\prime}(t)\right)^{2}}-c(t) p^{\prime}(t-\tau(t)) .
$$

Let $\psi \in C(\sigma)$ be fixed and define

$S=\{\phi \in C([m(\sigma), \infty), R): \phi(t)=\psi(t)$, if $t \in[m(\sigma), \sigma], \phi(t) \rightarrow 0$, as $t \rightarrow \infty$, and $\phi$ is bounded $\}$ with metric $\rho(\xi, \eta)=\sup _{t \geq \sigma}|\xi(t)-\eta(t)|$. Then $S$ is a complete metric space. Define the mapping $Q: S \rightarrow S$ by $(Q \phi)(t)=\psi(t)$ for $t \in[m(\sigma), \sigma]$ and for $t \geq \sigma$

$$
(Q \varphi)(t)=\sum_{i=1}^{5} I_{i}(t)
$$




$$
\begin{aligned}
& I_{1}(t)=\left(\psi(\sigma)-\frac{p(\sigma-\tau(\sigma))}{p(\sigma)} \frac{c(\sigma)}{1-\tau^{\prime}(\sigma)} \psi(\sigma-\tau(\sigma))-\int_{\sigma-\tau(\sigma)}^{\sigma} h(s) \psi(s) d s\right) e^{-\int_{\sigma}^{t} h(u) d u} \\
& I_{2}(t)=\frac{p(t-\tau(t))}{p(t)} \frac{c(t)}{1-\tau^{\prime}(t)} \varphi(t-\tau(t)) \\
& I_{3}(t)=\int_{t-\tau(t)}^{t} h(s) \varphi(s) d s \\
& I_{4}(t)=\int_{\sigma}^{t} e^{-\int_{s}^{t} h(u) d u}\left(-\beta(s)+h(s-\tau(s))\left(1-\tau^{\prime}(s)\right)-r(s)\right) \varphi(t-\tau(t)) d s \\
& I_{5}(t)=\int_{\sigma}^{t} e^{-\int_{s}^{t} h(u) d u} h(s)\left(\int_{s-\tau(s)}^{s} h(u) \varphi(u) d u\right) d s
\end{aligned}
$$

Next, we prove $Q \phi \in S$. Let be small and $\phi \in S$, then there are constants $\delta, L>0$ such that $\|\psi\|<\delta$ and $\|\phi\|<L$. From assumption (6), we get

$$
|(Q \varphi)(t)| \leq\left(1+\int_{\sigma-\tau(\sigma)}^{\sigma}|h(u)| d u+\left|\frac{p(\sigma-\tau(\sigma))}{p(\sigma)} \frac{c(\sigma)}{1-\tau^{\prime}(\sigma)}\right|\right) \delta K(\sigma)+\alpha L \leq 2 \delta K(\sigma)+\alpha L,
$$

where $K(\sigma)=\sup _{t \geq \sigma}\left\{e^{-\int_{\sigma}^{t} h(s) d s}\right\}$. Since $\lim _{t \rightarrow \infty} \int_{\sigma}^{t} h(u) d u=\infty$ implies $K(\sigma)<\infty$, then we get that $Q \phi$ is bounded. It is clear that $Q \phi$ is continuous. We now prove that $Q \phi(t) \rightarrow 0$ as $t \rightarrow \infty$. Obviously $I_{i}(t) \rightarrow 0$ for $i=1,2,3$ since $\int_{\sigma}^{t} h(u) d u \rightarrow \infty, t-\tau(t) \rightarrow \infty$ and $\phi$ $(t) \rightarrow 0$ as $t \rightarrow \infty$. Next, we prove that $I_{4}(t) \rightarrow 0$ as $t \rightarrow \infty$. For $t-\tau(t) \rightarrow \infty$ and $\phi(t) \rightarrow$ 0 , we get that for any $\varepsilon>0$, there is a positive number $T_{1}>0$, such that $\phi(t-\tau(t))<\varepsilon$ for all $t \geq T_{1}$. Then

$$
\begin{aligned}
& \left|I_{4}(t)\right| \leq e^{-\int_{T_{1}}^{t} h(u) d u} \int_{\sigma}^{T_{1}} e^{-\int_{s}^{T_{1}} h(u) d u}\left|-\beta(s)+h(s-\tau(s))\left(1-\tau^{\prime}(s)\right)-r(s)\right||\varphi(t-\tau(t))| d s \\
& \quad+\int_{T_{1}}^{t} e^{-\int_{s}^{t} h(u) d u}\left|-\beta(s)+h(s-\tau(s))\left(1-\tau^{\prime}(s)\right)-r(s)\right||\varphi(t-\tau(t))| d s \\
& \leq \max _{t \geq m(\sigma)}|\varphi(t)| e^{-\int_{s}^{t} h(u) d u} \int_{\sigma}^{T_{1}} e^{-\int_{s}^{T_{1}} h(u) d u}\left|-\beta(s)+h(s-\tau(s))\left(1-\tau^{\prime}(s)\right)-r(s)\right| d s \\
& +\varepsilon \int_{T_{1}}^{t} e^{-\int_{s}^{t} h(u) d u}\left|-\beta(s)+h(s-\tau(s))\left(1-\tau^{\prime}(s)\right)-r(s)\right| d s \\
& \leq \alpha \max _{t \geq m(\sigma)}|\varphi(t)| e^{-\int_{s}^{t} h(u) d u}+\alpha \varepsilon<\varepsilon
\end{aligned}
$$

as $t$ is large enough. Similarly, we can prove that $I_{i}(t) \rightarrow 0$ for $i=5$. So we get that $(Q \phi)(t) \mid \rightarrow 0$ as $t \rightarrow \infty$ and hence $Q \phi \in S$. Now, it remains to show that $Q$ is a contraction mapping.

Let $\xi, \eta \in S$, then

$$
\begin{aligned}
|(Q \xi)(t)-(Q \eta)(t)| \leq & \left\{\left|\frac{p(t-\tau(t))}{p(t)} \frac{c(t)}{1-\tau^{\prime}(t)}\right|+\int_{t-\tau(t)}^{t}|h(s)| d s\right. \\
& +\int_{\sigma}^{t} e^{-\int_{s}^{t} h(u) d u}\left|-\beta(s)+h(s-\tau(s))\left(1-\tau^{\prime}(s)\right)-r(s)\right| d s \\
& \left.+\int_{\sigma}^{t} e^{-\int_{s}^{t} h(u) d u}|h(s)|\left(\int_{s-\tau(s)}^{s}|h(u)| d u\right) d s\right\}\|\xi-\eta\| \\
\leq & \alpha\|\xi-\eta\| .
\end{aligned}
$$

Therefore, $Q$ is a contraction mapping with contraction constant $\alpha<1$. By the contraction mapping principle, $Q$ has a unique fixed point $z$ in $S$ which is a solution of (8) with $z$ $(t)=\psi(t)$ on $[m(\sigma), \sigma]$ and $z(t)=z(t, \sigma, \psi)$ is bounded and tends to zero as $t \rightarrow \infty$. To obtain asymptotic stability, we need to show that the zero solution of (8) is stable. Let $\varepsilon$ $>0$ be given and choose $\delta>0$ such that $\delta<\varepsilon$ and $2 \delta K(\sigma)+\alpha \varepsilon<\varepsilon$. If $z(t)=z\left(t, t_{0}, \psi\right)$ is a 
solution of (8) with $\|\psi\|<\delta$, then $z(t)=(Q z)(t)$ as defined in (9). We claim that $|z(t)|<\varepsilon$ for all $t \geq \sigma$. It is clear that $|z(t)|<\varepsilon$ on $[m(\sigma), \sigma]$. If there exists $t_{0}>\sigma$ such that $\left|z\left(t_{0}\right)\right|=$ $\varepsilon$ and $|z(s)|<\varepsilon$ for $m(\sigma) \leq s<t_{0}$, then it follows from (9) that

$$
\begin{aligned}
\left|z\left(t_{0}\right)\right| \leq & \|\psi\|\left(1+\left|\frac{p(\sigma-\tau(\sigma))}{p(\sigma)} \frac{c(\sigma)}{1-\tau^{\prime}(\sigma)}\right|+\int_{\sigma-\tau(\sigma)}^{\sigma}|h(s)| d s\right) e^{-\int_{\sigma}^{t_{0}} h(u) d u} \\
& +\varepsilon\left\{\left|\frac{p(t-\tau(t))}{p(t)} \frac{c(t)}{1-\tau^{\prime}(t)}\right|+\int_{t-\tau(t)}^{t}|h(s)| d s\right. \\
& +\int_{\sigma}^{t} e^{-\int_{s}^{t} h(u) d u}\left|-\beta(s)+h(s-\tau(s))\left(1-\tau^{\prime}(s)\right)-r(s)\right| d s \\
& \left.+\int_{\sigma}^{t} e^{-\int_{s}^{t} h(u) d u}|h(s)|\left(\int_{s-\tau(s)}^{s}|h(u)| d u\right) d s\right\} \leq 2 \delta K(\sigma)+\alpha \varepsilon<\varepsilon
\end{aligned}
$$

which contradicts that $\left|z\left(t_{0}\right)\right|=\varepsilon$. Then, $|z(t)|<\varepsilon$ for all $t \geq \sigma$, and the zero solution of (8) is stable.

Thus, the zero solution of (8) is asymptotically stable, and hence the zero solution of (1) is asymptotically stable. The proof is complete.

Remark 2. Let $p(t) \equiv 1$, then Theorem 3.1 is Theorem $B$ on sufficient conditions.

Theorem 3.2. Let $\tau(t)$ be twice differentiable and $\tau^{\prime}(t) \neq 1$ for all $t \in[m(\sigma), \infty)$. Suppose that (i)-(iii) in Theorem 3.1 hold. If there exist $\lambda(t)$ as defined in Definition 2.2 and constant $\gamma>0$ such that $\limsup _{t \rightarrow \infty} \frac{\log p(t)}{\log \lambda(t)} \leq-\gamma$, then the zero solution of (1) is $\lambda$ stable.

Proof. By combining Theorem 3.1 and $\limsup _{t \rightarrow \infty} \frac{\log p(t)}{\log \lambda(t)} \leq-\gamma$, we show that the zero solution of (1) is $\lambda$-stable.

Similar to Theorems 3.1 and 3.2, we consider the stability of the generalized linear neutral equations with variable delays. The proof is omitted for similarity.

$$
x^{\prime}(t)=-a(t) x(t)-\sum_{j=1}^{N} b_{j}(t) x\left(t-\tau_{j}(t)\right)+\sum_{j=1}^{M} c_{j}(t) x^{\prime}\left(t-\tau_{j}(t)\right)
$$

Theorem 3.3. Let $\tau_{j}(t)$ be twice differentiable and $\tau_{j}^{\prime}(t) \neq 1$ for all $t \in\left[m_{j}(\sigma), \infty\right)$. Suppose that

(i) there exist continuous functions $h_{j}:\left[m_{j}(\sigma), \infty\right) \rightarrow R$ such that $\lim _{t \rightarrow \infty} \int_{\sigma}^{t} H(u) d u=\infty$;

(ii) there exists a bounded function $p:[\bar{m}(\sigma), \infty) \rightarrow(0, \infty)$ with $p(\sigma)=1$ such that $p^{\prime}(t)$ exists on $[\bar{m}(\sigma), \infty)$;

(iii) there exists a constant $\alpha \in(0,1)$ such that for $t \geq \sigma$

$$
\begin{aligned}
& \sum_{j=1}^{N \vee M}\left|\frac{p\left(t-\tau_{j}(t)\right)}{p(t)} \frac{c_{j}(t)}{1-\tau_{j}^{\prime}(t)}\right|+\sum_{j=1}^{N \vee M} \int_{t-\tau_{i}(t)}^{t}\left|h_{j}(s)-A_{m, j}(s)\right| d s \\
& +\sum_{j=1}^{N \vee M} \int_{\sigma}^{t} e^{-\int_{s}^{t} H(u) d u}\left|-\beta_{j}(s)+\left[h_{j}\left(s-\tau_{j}(s)\right)-A_{m, j}\left(s-\tau_{j}(s)\right)\right]\left(1-\tau_{j}^{\prime}(s)\right)-r_{j}(s)\right| d s \\
& +\sum_{j=1}^{N \vee M} \int_{\sigma}^{t} e^{-\int_{s}^{t} H(u) d u}|H(s)|\left(\int_{s-\tau_{i}(s)}^{s}\left|h_{j}(u)-A_{m, j}(u)\right| d u\right) d s \leq \alpha,
\end{aligned}
$$




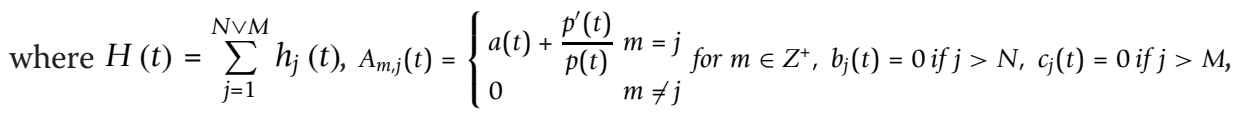

$$
\beta_{j}(t)=\frac{b_{j}(t) p\left(t-\tau_{j}(t)\right)+c_{j}(t) p^{\prime}\left(t-\tau_{j}(t)\right)-p^{\prime}(t)}{p(t)}
$$

and

$$
r_{j}(t)=\frac{H(t) c_{j}(t) p(t) p\left(t-\tau_{j}(t)\right)+c_{j}^{\prime}(t) p\left(t-\tau_{j}(t)\right)}{p^{2}(t)\left(1-\tau_{j}^{\prime}(t)\right)}+\frac{c_{j}(t) p\left(t-\tau_{j}(t)\right) \tau^{\prime \prime}{ }_{j}(t)}{p(t)\left(1-\tau^{\prime} j(t)\right)^{2}}-c_{j}(t) p^{\prime}\left(t-\tau_{j}(t)\right) .
$$

(1) Then the zero solution of (2) is asymptotically stable.

(2) If there exist $\lambda(t)$ as defined in definition 2.2 and constant $\gamma>0$ such that $\limsup _{t \rightarrow \infty} \frac{\log p(t)}{\log \lambda(t)} \leq-\gamma$, then the zero solution of (2) is $\lambda$ - stable.

Remark 3. Similar to argument in [20]. The method in this article can be extended to the following nonlinear neutral differential equations with variable delays:

$$
x^{\prime}(t)=-a(t) x(t)+b(t) g(x(t-\tau(t)))+c(t) x^{\prime}(t-\tau(t))
$$

where $g$ is supposed to be a locally Lipschitz such that $|g(x)-g(y)|<|x-y|$ whenever $\mid$ $x|| y \mid, \leq L$ for some $L>0$ and $g(0)=0$.

\section{Examples}

Example 1. Consider the neutral differential equation with variable delays

$$
x^{\prime}(t)=-b(t) x(t-\tau(t))+c(t) x^{\prime}(t-\tau(t))
$$

for $t \geq 0$, where $c(t)=\sin ^{2}(t), \tau(t)=\frac{\pi}{2}, \quad b(t) \quad$ satisfies $\left|-\beta(t)+h(t-\tau(t))\left(1-\tau^{\prime}(t)\right)-r(t)\right| \leq \frac{0.01}{3+t}$ with $h(t)=\frac{0.05}{3+t}$ and $p(t)=1+\sin ^{2}(t)$. Then the zero solution of $(10)$ is asymptotically stable.

Proof. By choosing $h(t)=\frac{0.05}{3+t}$ and $p(t)=1+\sin ^{2}(t)$ in Theorem 3.1, we have

$$
\begin{aligned}
& \left|\frac{p(t-\tau(t))}{p(t)} \frac{c(t)}{1-\tau^{\prime}(t)}\right|=\frac{1+\sin ^{2}\left(t-\frac{\pi}{2}\right)}{1+\sin ^{2} t} \sin ^{2} t=\frac{2-\sin ^{2}(t)}{1+\sin ^{2} t} \sin ^{2} t \leq 0.536, \\
& \int_{t-\tau(t)}^{t}|h(s)| d s=\int_{t-\tau(t)}^{t} \frac{0.05}{3+s} d s=0.05 \ln \frac{3+t}{3+t-\frac{\pi}{2}} \leq 0.05, \\
& \int_{0}^{t} e^{-\int_{s}^{t} h(u) d u}\left|-\beta(s)+h(s-\tau(s))\left(1-\tau^{\prime}(s)\right)-r(s)\right| d s=\int_{0}^{t} e^{-\int_{s}^{t} \frac{0.05}{3+s} d u} \frac{0.01}{3+s} d s \leq 0.2
\end{aligned}
$$

and

$$
\begin{aligned}
\int_{0}^{t} e^{-\int_{s}^{t} h(u) d u}|h(s)|\left(\int_{s-\tau(s)}^{s}|h(u)| d u\right) d s & =\int_{0}^{t} e^{-\int_{s}^{t} \frac{0.05}{3+s} d u} \frac{0.05}{3+s}\left(\int_{s-\tau(s)}^{s} \frac{0.05}{3+u} d u\right) d s \\
& \leq 0.05 \int_{0}^{t} e^{-\int_{s}^{t} \frac{0.05}{3+s} d u} \frac{0.05}{3+s} d s \leq 0.05 .
\end{aligned}
$$

Therefore, $\alpha=0.536+0.05+0.2+0.05=0.836<1$. Moreover, $1 \leq p(t) \leq 2$ and all the conditions of Theorem 3.1 hold. So, the zero solution of (10) is asymptotically stable by Theorem 3.1. $\square$ 
Remark 4. Consider equation with $a, b, c$ defined as in Example 1. Then

$$
\left|\frac{c(t)}{1-\tau^{\prime}(t)}\right|=\left|\sin ^{2} t\right|=1 \text { when } t=k \pi+\frac{\pi}{2} \text { for } k=0,1,2, \ldots
$$

This implies that contraction conditions (3), (4), and (5) do not hold. Thus, Theorems $A, B$, and $C$ all cannot be applied to Equation 10.

Example 2. Consider the neutral differential equation with variable delays

$$
x^{\prime}(t)=-a(t) x(t)+c(t) x^{\prime}(t-\tau(t))
$$

for $t \geq 0$, where $c(t)=0.2, \tau(t)=0.1 t, a(t)=\frac{2}{1+t}$.Then the zero solution of $(11)$ is asymptotically stable and also polynomially stable.

Proof. By choosing $h(t)=\frac{2.3}{1+t}$ and $p(t)=\frac{1.2}{1+t}$ in Theorem 3.3 with $N=1, M=1$ and $b_{1}$ $(t) \equiv 0$, we have

$$
\begin{aligned}
& \left|\frac{p(t-\tau(t))}{p(t)} \frac{c(t)}{1-\tau^{\prime}(t)}\right|=\frac{1+t}{1+t-0.1 t} \frac{0.2}{0.9} \leq 0.25, \\
& \int_{t-\tau(t)}^{t}\left|h(s)-a(s)-\frac{p^{\prime}(s)}{p(s)}\right| d s=\int_{t-0.1 t}^{t} \frac{1.3}{1+s} d s=1.3 \ln \frac{10}{9} \leq 0.14 \\
& \int_{0}^{t} e^{-\int_{s}^{t} h(u) d u}|h(s)|\left(\int_{s-\tau(s)}^{s}\left|h(u)-a(u)-\frac{p^{\prime}(u)}{p(u)}\right| d u\right) d s \\
& =\int_{0}^{t} e^{-\int_{s}^{t} \frac{2.3}{1+s} d u} \frac{2.3}{1+s}\left(\int_{s-\tau(s)}^{s} \frac{1.3}{1+u} d u\right) d s \leq 0.14 \int_{0}^{t} e^{-\int_{s}^{t} \frac{2.3}{1+s} d u} \frac{2.3}{1+s} d s \leq 0.14
\end{aligned}
$$

and

$$
\begin{aligned}
& \int_{0}^{t} e^{-\int_{s}^{t} h(u) d u}\left|-\beta(s)+\left[h(s-\tau(s))-a(s-\tau(s))-\frac{p^{\prime}(s-\tau(s))}{p(s-\tau(s))}\right]\left(1-\tau^{\prime}(s)\right)-r(s)\right| d s \\
& =\int_{0}^{t} e^{-\int_{s}^{t} \frac{2.3}{1+s} d u}\left|-\left(\frac{1}{1+s}-\frac{0.2(1+s)}{(1+0.9 s)^{2}}\right)+0.9 \frac{1.3}{1+0.9 s}-\left(\frac{0.46}{0.9} \frac{1}{1+0.9 s}+0.2 \frac{1.2}{(1+0.9 s)^{2}}\right)\right| d s \\
& =\int_{0}^{t} e^{-\int_{s}^{t} \frac{2.3}{1+s} d u}\left|\frac{0.1 s+(1+s)\left(0.17-\frac{0.46}{0.9}+0.2 \frac{1+s}{1+0.9 s}-\frac{0.24}{1+0.9 s}\right)}{(1+s)(1+0.9 s)}\right| d s \\
& \leq \int_{0}^{t} e^{-\int_{s}^{t} \frac{2.3}{1+s} d u} \frac{0.1 s+(1+s)\left(\left|0.17-\frac{0.46}{0.9}\right|+0.2 \frac{|s-0.2|}{1+0.9 s}\right)}{(1+s)(1+0.9 s)} d s \\
& \leq \int_{0}^{t} e^{-\int_{s}^{t} \frac{2.3}{1+s} d u} \frac{5}{9(1+s)} d s \leq 0.25 \text {. }
\end{aligned}
$$

Therefore, $\alpha=0.25+0.14+0.14+0.25=0.78<1$. (i)-(iii) in Theorem 3.3 hold. So, the zero solution of (11) is asymptotically stable by (1) of Theorem 3.3. Moreover, $p(t)=\frac{1.2}{1+s}$ and hence the zero solution of (11) is polynomially stable by (2) of Theorem 3.3 with $\lambda(t)=1+t$.

\section{Conclusion}

In this article, we study a class of the linear neutral differential equation with variable delays, several special cases of which have been studied in [2,17-19]. Some of the results, like Theorems A, B, and C, mainly dependent on the constraint $\left|\frac{c(t)}{1-\tau^{\prime}(t)}\right|<1$. But in many environments, the constraint is not satisfied. So, by employing an auxiliary 
function $p(t)$ on the contraction condition, we get new criteria for asymptotic stability of the zero solution by using the fixed point method and the differential inequality techniques which not only includes the results on sufficient part in [17-19], but also includes several equations that previously known related results can not be applied to. Another application of the method in this article is to obtain the decay rates of the solutions including exponential stability, polynomial stability, logarithmical stability, etc., in which only the exponential stability has been discussed by Luo $[13,14]$ and Zhou and Zhong [15] with the fixed points method. As the linear neutral differential equations like (2) and it's special cases are considered, the results of this article are new and they extend and improve previously known results.

\section{Acknowledgements}

The author sincerely thanks the anonymous reviewers for their careful reading and fruitful suggestions to improve the quality of the manuscript. This article was partially supported by NSFC (No. 11001173).

\section{Author details}

${ }^{1}$ College of Science, University of Shanghai for Science and Technology, Shanghai, 200093, China ${ }^{2}$ Department of Mathematics, Shanghai Jiaotong University, Shanghai 200240, China

\section{Competing interests}

The authors declare that they have no competing interests.

Received: 17 June 2011 Accepted: 31 October 2011 Published: 31 October 2011

\section{References}

1. Becker, LC, Burton, TA: Stability, fixed points and inverses of delays. Proc R Soc Edinb Sect A. 136, 245-275 (2006). doi:10.1017/S0308210500004546

2. Burton, TA: Fixed points, stability, and exact linearization. Nonlinear Anal. 61, 857-870 (2005). doi:10.1016/j. na.2005.01.079

3. Burton, TA: Fixed points, Volterra equations, and Becker's resolvent. Acta Math Hungar. 108, 261-281 (2005). doi:10.1007/s10474-005-0224-9

4. Burton, TA: Fixed points and stability of a nonconvolution equation. Proc Am Math Soc. 132, 3679-3687 (2004). doi:10.1090/S0002-9939-04-07497-0

5. Burton, TA: Perron-type stability theorems for neutral equations. Nonlinear Anal. 55, 285-297 (2003). doi:10.1016/50362546X(03)00240-2

6. Burton, TA: Integral equations, implicit functions, and fixed points. Proc Am Math Soc. 124, 2383-2390 (1996). doi:10.1090/S0002-9939-96-03533-2

7. Burton, TA: Tetsuo Furumochi, Krasnoselskii's fixed point theorem and stability. Nonlinear Anal. 49, 445-454 (2002). doi:10.1016/S0362-546X(01)00111-0

8. Burton, TA, Zhang, B: Fixed points and stability of an integral equation: nonuniqueness. Appl Math Lett. 17, 839-846 (2004). doi:10.1016/j.aml.2004.06.015

9. Burton, TA: Stability by fixed point theory or Liapunov's theory: a comparison. Fixed Point Theory. 4, 15-32 (2003)

10. Zhang, B: Fixed points and stability in differential equations with variable delays. Nonlinear Anal. 63, e233-e242 (2005). doi:10.1016/j.na.2005.02.081

11. Sakthivel, R, Luo, J: Asymptotic stability of nonlinear impulsive stochastic differential equations. Stat Probab Lett. 79, 1219-1223 (2009). doi:10.1016/j.spl.2009.01.011

12. Sakthivel, R, Luo, J: Asymptotic stability of impulsive stochastic partial differential equations with infinite delays. J Math Anal Appl. 356, 1-6 (2009). doi:10.1016/j.jmaa.2009.02.002

13. Luo, J: Fixed points and exponential stability of mild solutions of stochastic partial differential equations with delays. J Math Anal Appl. 342, 753-760 (2008). doi:10.1016/j.jmaa.2007.11.019

14. Luo, J: Fixed points and exponential stability for stochastic Volterra-Levin equations. J Math Anal Appl. 234, 934-940 (2010)

15. Zhou, X, Zhong, S: Fixed point and exponential p-stability of neutral stochastic differential equations with multiple delays. Proceedings of the 2010 IEEE International Conference on Intelligent Computing and Intelligent Systems, ICIS 1 238-242 (2010). art. no. 5658577

16. Pinto, $\mathrm{M}$, Seplveda, D: $\mathrm{H}$-asymptotic stability by fixed point in neutral nonlinear differential equations with delay. Nonlinear Anal. 74(12):3926-3933 (2011). doi:10.1016/j.na.2011.02.029

17. Raffoul, YN: Stability in neutral nonlinear differential equations with functional delays using fixed-point theory. Math Comput Model. 40, 691-700 (2004). doi:10.1016/j.mcm.2004.10.001

18. Jin, C, Luo, J: Fixed points and stability in neutral differential equations with variable delays. Proc Am Math Soc. 136, 909-918 (2008)

19. Ardjouni, A, Djoudi, A: Fixed points and stability in linear neutral differential equations with variable delays. Nonlinear Anal. 74, 2062-2070 (2011). doi:10.1016/j.na.2010.10.050 
20. Djoudi, A, Khemis, R: Fixed point techniques and stability for neutral nonlinear differential equations with unbounded delays. Georg Math J. 13(1):25-34 (2006)

doi:10.1186/1687-1847-2011-48

Cite this article as: Zhao: New criteria for stability of neutral differential equations with variable delays by fixed points method. Advances in Difference Equations 2011 2011:48.

Submit your manuscript to a SpringerOpen ${ }^{\circ}$ journal and benefit from:

- Convenient online submission

- Rigorous peer review

- Immediate publication on acceptance

- Open access: articles freely available online

- High visibility within the field

- Retaining the copyright to your article

Submit your next manuscript at $\gg$ springeropen.com 